\title{
The Protease Inhibitor Era: An Opportunity to Improve the Quality of Care
}

\author{
Andrew J. Muir
}

Published online: 18 March 2011

(C) Springer Science+Business Media, LLC 2011

Treatment for chronic hepatitis $\mathrm{C}$ virus (HCV) infection is expected to take a tremendous step forward this year when boceprevir and telaprevir undergo review by the United States Food and Drug Administration [1,2]. These protease inhibitors will be administered in combination with the current standard of care of peginterferon alfa and ribavirin and will mark the first change in treatment in a decade. The addition of a direct acting antiviral (DAA) medication raises a number of new issues for providers of $\mathrm{HCV}$ treatment. These medications bring the excitement of improved response rates but also a number of challenges. They will be delivered in a response guided therapy algorithm, and they carry new adverse events and the threat of resistance.

In this issue, Gaglio and colleagues [3] present a survey to assess the current knowledge of members of the American Gastroenterological Association and American Association for the Study of Liver Diseases regarding DAA medications and their attitudes regarding the upcoming treatment regimens. Of the 10,082 surveys sent out, the response rate was $21 \%$. Although we can debate the adequacy of the response rate, this sample of 1,320 current treatment providers offers insight into the perceptions of many future providers of these medication regimens. A major concern in our field has been that the added complexity of $\mathrm{HCV}$ treatment with protease inhibitors will lead some current providers to cease offering treatment. Among this sample in which $85 \%$ provide their own treatment currently, $81 \%$ plan to offer treatment in the DAA era,

\section{A. J. Muir $(\bowtie)$}

Division of Gastroenterology, Duke Clinical Research Institute, Duke University School of Medicine, P.O. Box 17969, Durham, NC 27715, USA

e-mail: muir0002@mc.duke.edu while the proportion referring to a $\mathrm{HCV}$ specialist only increased from 6 to $10 \%$. Although these numbers are statistically significant, the survey demonstrates that many current $\mathrm{HCV}$ providers plan to continue treatment in the future, and this sample does not provide evidence of a large exodus from HCV treatment.

The next question is if these providers of HCV treatment are prepared for the new complexities of these triple therapy combinations. Gaglio and colleagues raise concerns about the $3 \%$ of respondents with minimal knowledge of DAA agents given that $59 \%$ of these providers report that they plan to offer these treatments in the future. The $77 \%$ of respondents who were aware or very aware of the DAA agents but had no participation in clinical trials will also need significant education and support in their initial phase of experience with these medications. These regimens will come with new treatment algorithms and stopping rules. Providers will need to understand viral resistance, including prevention strategies, monitoring requirements, and implications for treatment. Both boceprevir and telaprevir regimens lead to increased anemia, and patients receiving telaprevir need to be monitored for the development of a rash that requires careful monitoring and discontinuation of medication if progressive $[1,2]$. There is a natural learning curve to new treatments, and provider enthusiasm for the improved response rates could sour if poor outcomes occur from adverse events.

Gaglio and colleagues conclude that "extensive education of all future prescribers of DAA agents will be required to ensure successful use of these therapies". Historically, new therapies in our field have been the focus of industry-sponsored talks by thoughtful leaders and continuing medical education programs. Recently, these strategies have fallen out of favor given their failure to demonstrate improvements in the quality of care [4]. 
Instead, leaders in education have called for strategies within a continuing professional development framework with opportunities for self-assessment or external validation in addition to curriculum. The practice improvement modules offered by the American Board of Internal Medicine for maintenance of certification are examples of this approach. At this critical juncture in the care of $\mathrm{HCV}$, this type of strategy would seem warranted to improve the quality of care for patients with HCV infection.

Many of the key elements for this type of strategy are already present. In the last several years, HCV treatment has been identified as a priority by the Centers for Medicare and Medicaid Services, which has included a number of quality indicators in HCV care in the Physician Quality Reporting Initiative. The initial findings, however, highlight the concerns about the quality of care. In a recent retrospective cohort study from a nationwide US health insurance company research database with 10,385 patients with $\mathrm{HCV}$, only $18.5 \%$ of patients received all recommended care according to well established guidelines [5]. Only $21.5 \%$ of patients received hepatitis A vaccination. In evaluating patients receiving treatment, $\mathrm{HCV}$ genotype was performed in $79 \%$ of patients. HCV RNA was performed prior to treatment in $62.6 \%$ and at the 12-week stopping rule time point in $60.3 \%$. This glance at the care provided to this large group of patients with $\mathrm{HCV}$ demonstrates suboptimal care.

As we move towards treatment with the DAA regimens, it would be important for patients to know which practices or individuals will provide the best care with the DAA agents. The survey by Gaglio and colleagues offers distinctions among academic/private practices and experience in clinical trials, but it is not clear that these factors will predict treatment success. Previous studies found that studies in community settings achieved similar results to phase 3 trials of peginterferon alfa and ribavirin [6]. One could argue that community practices that elect to participate in clinical trials are not representative of all community practices and may have more experience but also more staff and infrastructure to support the clinical trial participants. Rather than a designation of private practice or academic, patients should seek practices that have significant experience with HCV treatment and have achieved excellent outcomes. Our anecdotal experience as an academic center seeing referrals from the community supports the notion that HCV therapy is delivered at a broad range of quality levels. Too many patients abort therapy and appear to have received little education in side effect management, and the well described stopping rules from our practice guidelines are not routinely followed [7]. At the same time, there are private practices in our region with no experience in the clinical trials with these agents, and yet there is reason to be confident that they will do very well with these new treatments. These practices have a tradition of high quality care and have invested in midlevel providers or nurse clinicians to offer $\mathrm{HCV}$ treatment. A recent study of $\mathrm{HCV}$ treatment within the Veterans Affairs medical centers evaluated completion rates of $\mathrm{HCV}$ treatment and also addressed predictors of successful treatment [8]. They found that sites that initiated more than 200 patients per year on treatment were much more likely to have patients complete treatment (odds ratio 1.87, 95\% confidence intervals 1.56-2.24).

As the protease inhibitors approach our clinics, are we worried treatment is going to become too complex? Or are we worried that more patients coming forward for treatment with these improved response rates will highlight the variable quality of HCV care? The scenario of the endoscopy unit nurse providing $\mathrm{HCV}$ treatment support during his or her spare time is not adequate. This care is best delivered by dedicated teams of physicians with midlevel providers and/or nurse clinicians. Practices with professional commitment to $\mathrm{HCV}$ care do this best, and we need to provide them the education and resources to be successful. An approach to continuing medical education that incorporates episodic learning with external validation of quality indicators would be one way to demonstrate this commitment. We need to nurture and identify these high quality practices and guide patients towards them if we hope to improve the overall quality of $\mathrm{HCV}$ care.

\section{Key Points}

- Protease inhibitors bring improved response rates but new challenges

- PQRI data: overall, HCV treatment quality is suboptimal

- HCV education requires ongoing learning plus assessment of quality indicators

- Patients should seek HCV care from experienced practices with excellent outcomes

\section{References}

1. Kwo PY, Lawitz EJ, McCone J, et al. Efficacy of boceprevir, an NS3 protease inhibitor, in combination with peginterferon alfa- $2 b$ and ribavirin in treatment-naive patients with genotype 1 hepatitis C infection (SPRINT-1): An open-label, randomised, multicentre phase 2 trial. Lancet. 2010;376:705-716.

2. McHutchison JG, Everson GT, Gordon SC, et al. Telaprevir with peginterferon and ribavirin for chronic HCV genotype 1 infection. N Engl J Med. 2009;360:1827-1838.

3. Gaglio PJ, Moss N, McGaw C, Reinus J. Direct-acting antiviral therapy for hepatitis C: attitudes regarding future use. Dig Dis Sci (Epub ahead of print). doi: 10.1007/s10620-011-1604-3. 
4. Bellande BJ, Winicur ZM, Cox KM. Urgently needed: A safe place for self-assessment on the path to maintaining competence and improving performance. Acad Med. 2010;85:16-18.

5. Kanwal F, Schnitzler M, Bacon BR, Hoang T, Buchanan PM, Asch SM. Quality of care in patients with chronic hepatitis C virus infection: A cohort study. Ann Intern Med. 2010;153:231-239.

6. Marotta P, Hueppe D, Zehnter E, Kwo P, Jacobson I. Efficacy of chronic hepatitis $\mathrm{C}$ therapy in community-based trials. Clin Gastroenterol Hepatol. 2009;7:1028-1035.
7. Ghany MG, Strader DB, Thomas DL, Seeff LB. Diagnosis, management, and treatment of hepatitis C: An update. Hepatology. 2009;49:1335-1374.

8. Butt A, McGinnis KA, Skanderson M, Justice AC. Hepatitis C treatment completion rates in routine clinical care. Liver Int. 2010;30:240-250. 